\title{
Staphylococcus aureus endocarditis presenting as acute scrotal pain
}

\section{Watanakunakorn}

Infectious Disease Section, Department of Internal Medicine, St. Elizabeth Hospital Medical Center, Youngstown, Ohio and Northenstern Ohio Universities College of Medicine, Rootstown, Ohio, USA.

\begin{abstract}
Summary: A previously healthy 17 year old boy had Staphylococcus aureus endoearditis presenting as acute scrotal pain. There was no trauma or evidence of scrotal or epididymal infection. The pain subsided after therapy for endocarditis was started.
\end{abstract}

\section{Introduction}

There are many salient features associated with Staphylococcus aureus endocarditis. ${ }^{1}$ Many patients with $S$. aureus endocarditis have severe shoulder pain or low back pain as a presenting symptom..$^{2-5}$ I recently saw a young man with $S$. aureus endocarditis presenting with acute right scrotal pain. I believe that this kind of presentation has not previously been reported.

\section{Case report}

A 17 year old previously healthy student complained of malaise, generalized body aches and weakness for two days. On the day of admission he had fever, a light headache and acute severe right scrotal pain, without trauma, dysuria or haematuria. He denied substance abuse. His temperature was $38.1^{\circ} \mathrm{C}$, pulse $96 / \mathrm{min}$, blood pressure $124 / 78 \mathrm{mmHg}$. There was a mitral systolic click but no murmur. The scrotum, testis and epididymis were all normal bilaterally. There were no petechiae. The leukocyte count was $12.8 \times 10^{9} / 1$ with $83 \%$ segmented forms and $1 \%$ band forms. Urinalysis was normal. Creatine phosphokinase was elevated at 330 units (normal 35-232). Three blood cultures were obtained.

He was seen by a urologist. A technetium scan of the scrotum was normal, demonstrating equal flow bilaterally. The next day one blood culture done on admission was reported as growing Gram-positive cocci. He was seen by a general surgeon whose diagnosis was of perforated retrocaecal appendicitis with referred pain to the right scrotum. An appen-

Correspondence: C. Watanakunakorn, M.D., F.A.C.P., F.C.C.P., St. Elizabeth Hospital Medical Center, 1044 Belmont Avenue, Youngstown, Ohio 44501-1790, USA. Accepted: 10 September 1986 dicectomy was performed with perioperative cefoxitin. The postoperative diagnosis was 'acute catarrhal appendicitis'.

On the third hospital day, when all three blood cultures taken on admission grew Gram-positive cocci, he was started on intravenous (i.v.) nafcillin. Three more blood cultures were done. All six blood cultures grew a penicillin-susceptible $S$. aureus (minimal inhibitory concentration $0.06 \mu \mathrm{g} / \mathrm{ml}$ ). When an infectious disease consultant saw the patient on the fourth hospital day, a grade $2 / 6$ systolic ejection murmur was heard at the second and third intercostal spaces in addition to the mitral systolic click. Nafcillin was discontinued and intravenous penicillin $G, 5$ million units i.v. every 6 hours, was started. The peak serum bactericidal titre was $1: 1024$ and trough $1: 32$ while on penicillin $G$. The scrotal pain gradually disappeared. The serum teichoic acid antibody titre was 1:16. After 3 weeks, the i.v. penicillin $G$ was changed to oral penicillin $V, 1$ gram every 4 hours (peak serum bactericidal titre $1: 128$ and trough $1: 32$ ), which was given for another 3 weeks. The patient's clinical course was uncomplicated. Echocardiogram showed mild aortic insufficiency and mitral valve prolapse.

\section{Discussion}

Acute scrotal pain as the chief presenting symptom of $S$. aureus endocarditis is unique and I believe has never previously been described. The basis of the scrotal pain is unclear, though it could have been due to emboli to the testis or scrotum, or it may have been of muscular origin, since the serum creatine kinase was elevated. Muscular pain is a well known symptom of endocarditis. ${ }^{2-5}$ The unnecessary appendicectomy 
could have been prevented if attention had been paid to the positive blood culture on the second hospital day. Endocarditis should be considered in any patient who has $S$. aureus septicaemia with no obvious portal of entry.

Another lesson to be learned from this case is that

\section{References}

1. Watanakunakorn, C., Tan, J.S. \& Phair, J.P. Some salient features of Staphylococcal aureus endocarditis. Am J Med 1973, 54: 473-481.

2. Holler, J.W. \& Pecora, J.S. Backache in bacterial endocarditis. NY State J Med 1970, 70: 1903-1905.

3. Churchill, M.A., Geraci, J.E. \& Hunder, G.G. Musculoskeletal manifestations of bacterial endocarditis. Ann Intern Med 1977, 87: 754-759. blood cultures should be part of routine investigation in any patient presenting with unexplained symptoms, severe pain or fever. Physicians should pay careful attention to the results of blood cultures and act accordingly.

4. Myers, O.L. \& Commerford, P.J. Musculoskeletal manifestations of bacterial endocarditis. Ann Rheum Dis 1977, $\vec{\circ}$ 36: 517-519.

5. Thomas, P.H., Allal, J., Bontoux, D. et al. Rheumatological manifestations of infective endocarditis. Ann Rheum Dis 1984, 43: 716-720. 